\title{
The relation between chemical abundances and kinematics of the Galactic disc with RAVE
}

\author{
C. Boeche ${ }^{1,2}$, C. Chiappini ${ }^{2}$, I. Minchev ${ }^{2}$, M. Williams ${ }^{2}$, M. Steinmetz ${ }^{2}$, S. Sharma ${ }^{3}$, G. Kordopatis ${ }^{4}$, \\ J. Bland-Hawthorn ${ }^{3}$, O. Bienaymé ${ }^{5}$, B. K. Gibson ${ }^{6,7}$, G. Gilmore ${ }^{4}$, E. K. Grebel ${ }^{1}$, A. Helmi ${ }^{8}$, U. Munari ${ }^{9}$, \\ J. F. Navarro ${ }^{10}$, Q. A. Parker ${ }^{11,12,13}$, W. Reid ${ }^{13}$, G. M. Seabroke ${ }^{14}$, A. Siebert ${ }^{4}$, A. Siviero ${ }^{15,2}$, F. G. Watson ${ }^{12}$, \\ R. F. G. Wyse ${ }^{16}$, and T. Zwitter ${ }^{17,18}$ \\ 1 Astronomisches Rechen-Institut, Zentrum für Astronomie der Universität Heidelberg, Mönchhofstr. 12-14, 69120 Heidelberg, \\ Germany \\ e-mail: corrado@ari.uni-heidelberg.de \\ 2 Leibniz-Institut für Astrophysik Potsdam (AIP), An der Sternwarte 16, 14482 Potsdam, Germany \\ Sydney Institute for Astronomy, University of Sydney, NSW 2006, Australia \\ Institute of Astronomy, University of Cambridge, Madingley Road, Cambridge CB3 0HA, UK \\ 5 Observatoire de Strasbourg, Université de Strasbourg, CNRS 11 rue de l'université, 67000 Strasbourg, France \\ 6 Jeremiah Horrocks Institute, University of Central Lancashire, Preston, PR1 2HE, UK \\ Monash Centre for Astrophysics, School of Mathematical Sciences, Monash University, Clayton, VIC, 3800, Australia \\ 8 Kapteyn Astronomical Institute, University of Groningen, PO Box 800, 9700 AV Groningen, The Netherlands \\ 9 INAF Osservatorio Astronomico di Padova, Via dell'Osservatorio 8, Asiago 36012, Italy \\ 10 University of Victoria, PO Box 3055, Station CSC, Victoria, BC V8W 3P6, Canada \\ 11 Department of Physics and Astronomy, Faculty of Sciences, Macquarie University, Sydney, NSW 2109, Australia \\ 12 Anglo-Australian Observatory, PO Box 296, Epping, NSW 1710, Australia \\ 13 Macquarie Research Centre for Astronomy, Astrophysics and Astrophotonics, Sydney, NSW 2109, Australia \\ 14 Mullard Space Science Laboratory, University College London, Holmbury St Mary, Dorking, RH5 6NT, UK \\ 15 Department of Physics and Astronomy, Padova University, Vicolo dellOsservatorio 2, 35122 Padova, Italy \\ 16 Department of Physics and Astronomy, Johns Hopkins University, 3400 North Charles Street, Baltimore, MD 21218, USA \\ 17 Faculty of Mathematics and Physics, University of Ljubljana, Jadranska 19, 1000 Ljubljana, Slovenia \\ 18 Center of Excellence SPACE-SI, Askerceva cesta 12, 1000 Ljubljana, Slovenia
}

Received 15 May 2012 / Accepted 13 February 2013

\section{ABSTRACT}

\begin{abstract}
Aims. We study the relations between stellar kinematics and chemical abundances of a large sample of RAVE giants in search of the selection criteria needed for disentangling different Galactic stellar populations, such as thin disc, thick disc and halo. A direct comparison between the chemo-kinematic relations obtained with our medium spectroscopic resolution data and those obtained from a high-resolution sample is carried out with the aim of testing the robustness of the RAVE data.

Methods. We selected a sample of 2167 giant stars with signal-to-noise per spectral measurements above 75 from the RAVE chemical catalogue and followed the analysis performed by Gratton and colleagues on 150 subdwarf stars spectroscopically observed at high resolution. We then used a larger sample of 9131 giants (with signal-to-noise above 60) to investigate the chemo-kinematical characteristics of our stars by grouping them into nine subsamples with common eccentricity $(e)$ and maximum distance achieved above the Galactic plane $\left(Z_{\max }\right)$.

Results. The RAVE kinematical and chemical data proved to be reliable by reproducing the results by Gratton et al. obtained with high-resolution spectroscopic data. We successfully identified three stellar populations that could be associated with the Galactic thin disc, a dissipative component composed mostly of thick-disc stars, as well as a component comprised of halo stars (presence of debris stars cannot be excluded). Our analysis, based on the $e-Z_{\max }$ plane combined with additional orbital parameters and chemical information, provides an alternative way of identifying different populations of stars. In addition to extracting canonical thick- and thin-disc samples, we find a group of stars in the Galactic plane $\left(Z_{\max }<1 \mathrm{kpc}\right.$ and $\left.0.4<e<0.6\right)$ that show homogeneous kinematics but differ in their chemical properties. We interpret this as a clear sign that some of these stars have experienced the effects of heating and/or radial migration, which have modified their original orbits. The accretion origin of such stars cannot be excluded.
\end{abstract}

Key words. Galaxy: abundances - Galaxy: evolution - Galaxy: kinematics and dynamics - Galaxy: structure

\section{Introduction}

The chemical enrichment of the Universe is one of the main thrusts of modern astrophysics, and the Milky Way (MW) can be seen as the Rosetta stone of this evolution. In particular, Galactic archeology, i.e., the combined study of kinematics and chemical composition of stars of different ages, has recently become one of the cornerstones for research in galaxy formation. The main goal in this new field is to reconstruct the formation history of our Galaxy by analysing its fossil chemical records and kinematical information. An important subproduct of this kind of study is also to provide observational constraints to models of galaxy formation in general. While there has been considerable progress in past years to reproduce realistic disc galaxies in cosmological 
simulations (e.g. Guedes et al. 2011; Brook et al. 2012a; Stinson et al. 2012), the outcome relies heavily on the advanced feedback models involving a considerable number of free parameters (Piontek \& Steinmetz 2011; Scannapieco et al. 2011) that need to be calibrated using empirically determined relations.

Two main paths have been taken in recent years in the field of Galactic archeology. On the one hand, high-resolution, high signal-to-noise (S/N) spectra of small/medium stellar samples ( 100-1000 stars) have been used to unveil the chemodynamical properties of the different galactic components (e.g., Gratton et al. 1996, 2000, 2003; Fuhrmann 1998, 2008; Adibekyan et al. 2011; Kordopatis et al. 2011b; Bensby \& Feltzing 2012, and references therein). Most of these studies have the disadvantage of being based on pre-selected samples defined according to strict kinematical criteria, hence suffering from biases that are difficult to quantify.

On the other hand, large spectroscopic surveys, such as the Geneva Copenhagen Survey (GCS, Nordström et al. 2004 16000 stars), the Sloan Extension for Galactic Understanding and Exploration ${ }^{1}$ (SEGUE, Yanny et al. 2009), and the RAdial Velocity Experiment (RAVE, Steinmetz at al. 2006; Zwitter et al. 2008; Siebert et al. 2011), take advantage of their large sample statistics (thus sampling a large parameter space) to compensate for the much less precise measurements of the abundances and stellar parameters, which are typical of medium/low spectral resolution (or photometry, in the case of GCS).

Most of the surveys quoted above aim at estimating the $[\alpha / \mathrm{Fe}]$ ratios of large samples of stars, which, to a first approximation, can be used as a proxy for the stellar relative age. This seems to be valid even when radial migration is present, see Schönrich \& Binney (2009b), Minchev et al. (2012d). The uncertainty in the $[\alpha / \mathrm{Fe}]$ ratios coming from the SEGUE abundance pipeline (Lee et al. 2008a,b) are around 0.2 dex, for $\mathrm{S} / \mathrm{N}$ above $\sim 20$. For the GCS, much less precise estimates based on Strömgren indices as proxy for $[\alpha / \mathrm{Fe}]$ were obtained (Casagrande et al. 2011).

Although the original goal of the RAVE survey is to obtain radial velocities for up to a million stars, its spectral measurements turned out to be very useful for chemo-dynamic analysis. Recently, the first results of the RAVE abundance pipeline have been published (the RAVE Chemical Catalogue - Boeche et al. 2011), showing that it is possible to measure up to seven individual elements from spectra with $\mathrm{S} / \mathrm{N}$ above 40 , and at least three of them from spectra with $S / N \sim 20$. The RAVE-chemical pipeline uncertainties for $[\alpha / \mathrm{Fe}]$ are similar to those of SEGUE around 0.2 dex.

A first study of the kinematic-abundance properties of the MW thin and thick discs using RAVE data has been carried out by Karataş \& Klement (2012), using results coming from the main RAVE pipeline (not from the chemical pipeline). The authors used a sample of $\sim 4000$ main-sequence stars (with $\log g>$ 3 ) from the second RAVE data release and classified the Galactic disc populations according to their distribution on the $V_{\text {rot }}-[\mathrm{M} / \mathrm{H}]$ plane, using the so-called $\mathrm{X}$ stellar population parameter defined by Schuster et al. (1993) ${ }^{2}$. Although the authors successfully reproduced the mean kinematic properties of the thin- and

\footnotetext{
1 For recent results with SEGUE data, see Cheng et al. (2012) ( 7000 stars), Schlesinger et al. (2012) ( 40000 G- and 23000 K-dwarfs), Bovy et al. (2012) ( 30000), Lee et al. (2011) ( 17000 stars), and Liu \& van de Ven (2012) ( 27 500 stars).

2 This parameter reflects the tendency for thin disc stars to be more metal-rich and faster rotating than stars in the thick disc, although it is clear that both components strongly overlap both in metallicity and Galactic rotation velocity.
}

thick-disc populations as compared to previous work (e.g., Veltz et al. 2008), their results should be taken with caution. Indeed, the metallicities and, in particular, the $[\alpha / \mathrm{Fe}]$ estimates coming from the RAVE main pipeline are not free of considerable errors and systematical effects (see a discussion in Siebert et al. 2011), which might be a problem for a classification method that relies on metallicity, such as the one adopted in Karataş \& Klement (2012).

Recently, the RAVE project has adopted a new pipeline (Kordopatis et al. 2011a; in prep.) to estimate the stellar parameters values such as effective temperature, gravity, and metallicity $\left(T_{\text {eff }}, \log g,[\mathrm{M} / \mathrm{H}]\right)$, which is free of most of the systematic errors cited before. Such values are required as input to the RAVE chemical pipeline, which estimates the chemical elemental abundances. To date, the RAVE-chemical catalogue constitutes the largest sample for which individual chemical abundances are available. Here, we exploit the RAVE chemical catalogue in the framework of abundance-kinematic correlations of stars in the Galaxy. We aim at disentangling the different Galactic stellar components, as well as at identifying stars which were accreted or experienced heating and/or radial migration along the evolution of our Galaxy. Possible processes that have been studied by means of $N$-body simulations include stellar diffusion driven by transient spiral arms (Sellwood \& Binney 2002; Roškar et al. 2008), by the interaction between spiral arms and the Galactic bar (Minchev \& Famaey 2010; Minchev et al. 2011; Brunetti et al. 2011) or by depositing stars into the Galactic discs by mergers (Abadi et al. 2003; Villalobos \& Helmi 2008; Di Matteo et al. 2012). Such a disc stirring can give rise to a number of phenomena in the chemo-kinematical properties of a galaxy, such as flattening in radial metallicity gradients (e.g., Schönrich \& Binney 2009a; Minchev et al. 2011; Pilkington et al. 2012) or extended stellar density profiles (e.g., Sánchez-Blázquez et al. 2009; Minchev et al. 2012b), and can have a profound impact on the way we interpret the abundance-kinematic correlations in our own Galaxy as recently discussed by Minchev et al. (2012d).

From the observational side, the main difficulty has been proper disentangling of the local thin and thick discs. No selection criterion is free of biases, be it kinematical or chemical. Some drawbacks of a separation based purely on the $[\alpha / \mathrm{Fe}]$ ratios in the framework of the SEGUE sample are discussed in Brauer et al. (in prep.) Recently Bovy et al. (2012) have even argued that there is no clear dichotomy between the thin and the thick discs, a result that contrasts to previous findings in similar studies (Veltz et al. 2008; Pasetto et al. 2012a,b). It thus seems that the best way to address this intricate problem is to study the properties of a sample spanning the largest possible parameter space, both in kinematics and in chemistry.

In the present work we make such an attempt by using the best-quality data of the RAVE abundance catalogue. Our two primary goals are a) to show that the RAVE data give results consistent with those found from higher resolution, higher $\mathrm{S} / \mathrm{N}$ analysis for the chemo-dynamical properties of nearby stars; and b) to properly investigate the abundance-kinematical properties of the thin and thick discs with a sample ten times larger, and which covers a much larger volume (from $\sim 100 \mathrm{pc}$ to $\sim 3-4 \mathrm{kpc}$ ) than the one adopted in the pioneering work of Gratton et al. (2003, hereafter G03).

The layout of the paper is as follows. In Sect. 2 we discuss our sample selection. Section 3 describes how the orbital parameters were computed. In Sect. 4 we carry out the analysis of the kinematic-abundance properties of the thin and thick discs divided according to the kinematic selection criteria adopted in 
C. Boeche et al.: The relation between chemical abundances and kinematics of the Galactic disc with RAVE
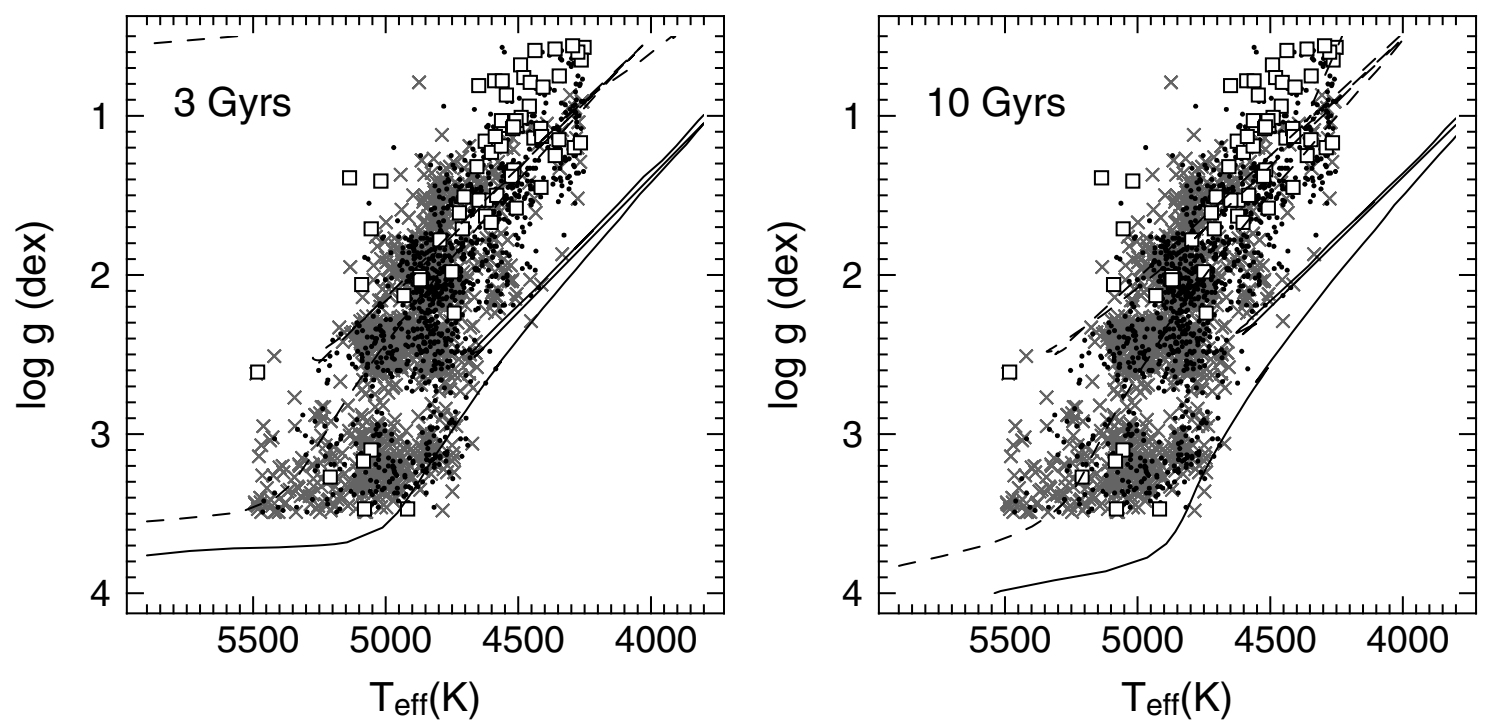

Fig. 1. $\log g$ versus $T_{\text {eff }}$ values for the SN75 RAVE sample (see text) with overplotted isochrones of 3 Gyr (left panel) and 10 Gyr (right panel) with metallicities $[\mathrm{M} / \mathrm{H}]=0.0 \mathrm{dex}$ (solid lines) and $[\mathrm{M} / \mathrm{H}]=-1.0 \mathrm{dex}$ (dashed lines). Black points, grey crosses, and open squares represent the thin disc, dissipative, and accretion components as defined in Sect. 4.

G03 and show that the RAVE abundance pipeline gives trustworthy results, despite the medium resolution of our spectra. In Sect. 5 we propose a different approach to the problem of disentangling different Galactic populations. Finally, discussion and conclusions are presented in Sect. 6.

\section{Data and sample selection}

This work makes use of the full suite of the RAVE data products, namely radial velocities, stellar parameters, distance estimates, and chemical abundances. Information on the radial velocities and proper motions come from Siebert et al. (2011), and distances have been computed following the recipe described in Burnett \& Binney (2011).

Recently, RAVE has adopted a new pipeline (Kordopatis et al. 2011a) for estimating the stellar parameters values. This pipeline makes use of the codes MATISSE (Recio-Blanco et al. 2006) and DEGAS (Bijaoui et al. 2012) optimized for the Ca II triplet region. These stellar parameters are adopted for the present work, and a more comprehensive description of the data will be presented in the next RAVE data release (Kordopatis et al., in prep.).

The present chemical abundances internal data release employed for this study contains elemental abundances for 245649 MW stars (for a detailed description of how these abundances are obtained, see Boeche et al. 2011). Roughly $10 \%$ of the RAVE stars have been observed more than once. To avoid using more than one estimation per star, for the re-observed stars we here adopt only the values derived from the spectrum with highest $\mathrm{S} / \mathrm{N}$.

For this first paper using the RAVE abundance catalogue, we have selected stars with the highest quality spectra and abundances. We additionally require that such stars cover a chemicalabundance range as large as possible (both in metallicity and $[\alpha / \mathrm{Fe}]$ ratios). To satisfy the first condition, we select spectra on which the code MATISSE converged to a single point of the parameter space $\left(T_{\text {eff }}, \log g,[\mathrm{M} / \mathrm{H}]\right)$, having high $\mathrm{S} / \mathrm{N}$ (at least $S / N=60$ ), which are well fit by the reconstructed spectra of the chemical pipeline $\left(\chi^{2}<1000\right)$ and with no continuum $\operatorname{defects}^{3}$ ( frac > 0.99). We also make certain that the selected spectra are not peculiar (classified as normal stars according to the three classification flags described in Matijevič et al. 2012). To satisfy the second condition, we only select cool giants and avoid dwarf stars. Indeed, owing to their weaker lines, the abundance measurements in dwarfs are more uncertain, especially for $[\mathrm{X} / \mathrm{H}]<-1.0$ dex. Conversely, for cool giants, thanks to their intense absorption lines, the RAVE abundance pipeline is able to measure chemical elements down to $[\mathrm{X} / \mathrm{H}]=-2.0$ dex. We exclude giants with $\log g<0.5$, to avoid any possible effects of the boundaries of the learning grid used for the automated parametrization (see Kordopatis et al., in prep.).

In the present work we consider the chemical abundances of Fe and $\alpha$ elements (the latter obtained as the average of $[\mathrm{Mg} / \mathrm{H}]$ and $[\mathrm{Si} / \mathrm{H}])$. These elements have the most precise abundances of all the elements included in the catalogue and, thus, are the most suitable ones for comparisons with the G03 work.

We decided to work with two samples of different $\mathrm{S} / \mathrm{N}$ : the main one contains only spectra with $S / N>75$ (the "SN75 sample") and the second one with $S / N>60$ (the "SN60 sample"), used when a better statistic is needed because it contains more stars ${ }^{4}$. Both samples satisfy the following criteria: i) giant stars with gravity $0.5<\log g<3.5$; ii) effective temperature $4000<T_{\text {eff }}(\mathrm{K})<5500$; iii) high quality data (spectra with $\chi^{2}<1000$ and frac $>0.99$ ). With these criteria, the SN75 and the SN60 samples count 2167 and 9131 stars, respectively.

These stellar parameter constraints are optimal for selecting cold giant stars from the RAVE sample and avoiding the hot giant stars of the horizontal branch. Figure 1 shows where our sample lies on the $T_{\text {eff- }} \log g$ plane. Also plotted are the isochrones of Marigo et al. (2008) for metallicities 0.0 and -1.0 dex and ages between 3 and 10 Gyr. Figure 2 shows the spatial distribution of our sample on the $x_{\mathrm{Gal}}-y_{\mathrm{Gal}}$ (Galactic disc) and $x_{\mathrm{Gal}}-z_{\mathrm{Gal}}$ (vertical direction) planes, where the Sun's location is indicated.

3 The frac parameter described in Boeche et al. (2011) gives the fraction of pixels that are non-defective and represents the goodness of the continuum fitting.

4 We verified that all the results of this work are valid for both samples. 

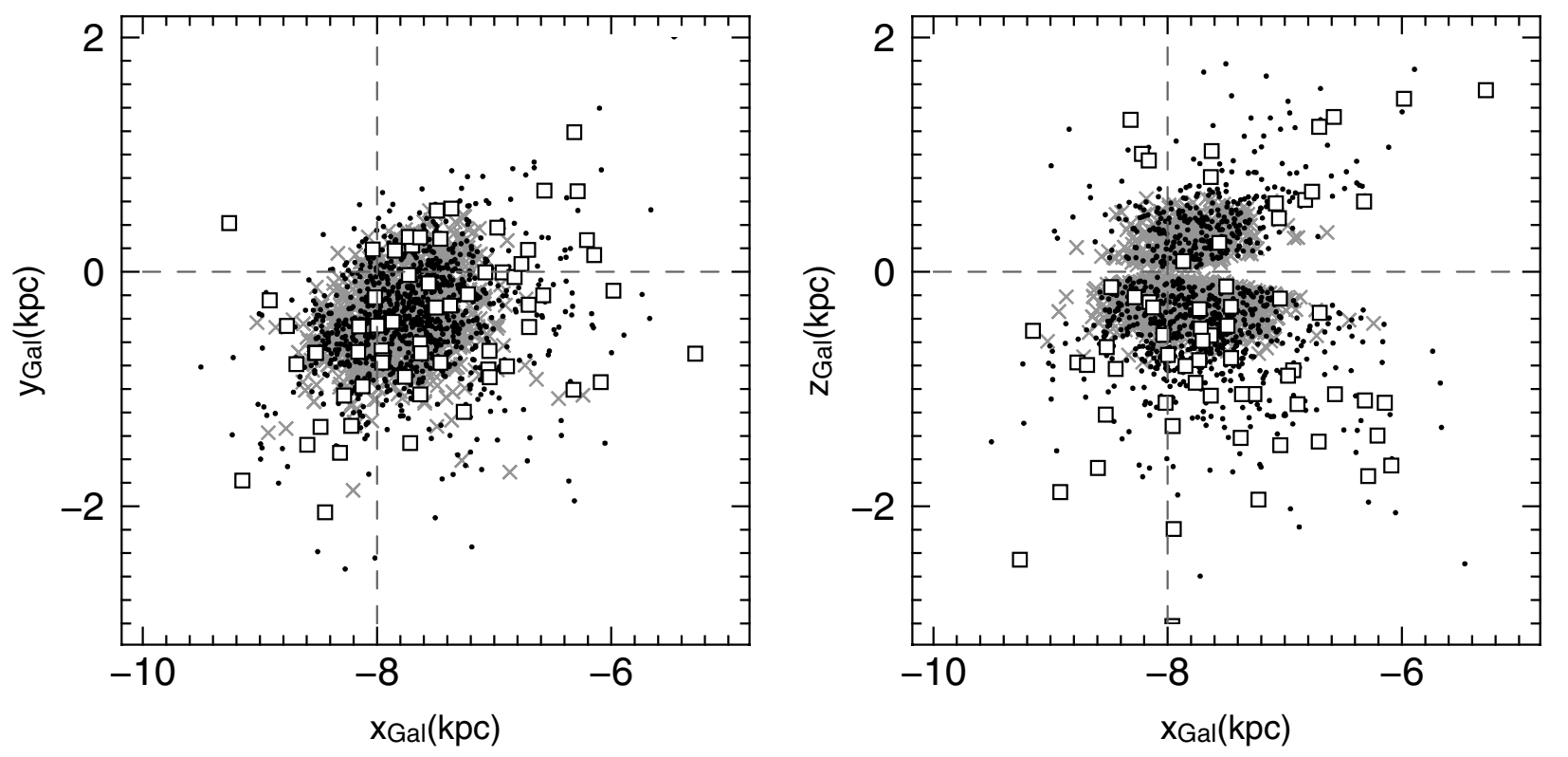

Fig. 2. Distribution of the SN75 RAVE sample in the $x_{\mathrm{Gal}}-y_{\mathrm{Gal}}$ and $x_{\mathrm{Gal}}-z_{\mathrm{Gal}}$ planes (left and right panels, respectively). The dashed lines cross at the Sun's position. Symbols as in Fig. 1.

\section{Orbital parameters}

To be self-consistent with the new stellar parameters adopted in this work (from the new RAVE pipeline - Kordopatis et al., in prep.), we recomputed the chemical abundances using the RAVE chemical pipeline (Boeche et al. 2011) and new distances for our stars by using the method described in Burnett \& Binney (2011) improved to take interstellar extinction and reddening into account (Binney et al., in prep.). We next computed the 3D space velocities $u, v$, and $w$ along the Galactic coordinates $x_{\mathrm{Gal}}, y_{\mathrm{Gal}}$ and $z_{\mathrm{Gal}}$. We corrected the velocities for the local standard of rest velocity as derived by Dehnen \& Binney (1998b). To obtain additional orbital parameters, we numerically integrated the orbits of stars by using the code NEMO (Teuben 1995), given their stellar distances and velocities. For the Galactic potential we adopted the model n.2 by Dehnen \& Binney (1998a), which assumes $R_{0}=8.0 \mathrm{kpc}$, circular velocity at the solar circle $v_{c}\left(R_{0}\right)=217 \mathrm{~km} \mathrm{~s}^{-1}$, and disc surface density $\Sigma=52.1 M_{\odot} \mathrm{pc}^{-2}$ (even when potentials n.1, 3, or 4 by Dehnen \& Binney 1998a were employed, the results did not change significantly). From the integrated Galactic orbits we extracted useful quantities, such as apocentre, $R_{\mathrm{a}}$, pericentre, $R_{\mathrm{p}}$, eccentricity, $e$, Galactic rotation velocity ${ }^{5} V_{\text {rot }}$, and the maximum vertical amplitude, $Z_{\max }$. The quantities $R_{\mathrm{a}}$ and $R_{\mathrm{p}}$ are the maximum and minimum distances from the Galactic centre a given star obtains during a revolution of $2 \pi$ radians around the Galactic centre, measured on the Galactic plane. Similarly, $Z_{\max }$ is the maximum altitude reached by a star along its orbit. The eccentricity is defined as $e=\left(R_{\mathrm{a}}-R_{\mathrm{p}}\right) /\left(R_{\mathrm{a}}+R_{\mathrm{p}}\right)$.

To estimate errors in the orbital parameters we followed the G03 method; i.e., we performed a Monte Carlo simulation. For each star we computed 100 times the orbital parameters by changing the Galactic coordinates $x_{\mathrm{Gal}}, y_{\mathrm{Gal}}, z_{\mathrm{Gal}}$ and the velocities $u, v, w$ every time, according to their estimated errors. From these 100 orbits we computed the standard deviation for each orbital parameter. Despite the long distance for some of our stars

\footnotetext{
5 Following the common use, the name "Galactic rotation velocity" refers here to the azimuthal velocity in Cilindrical coordinate system, computed as $V_{\text {rot }}=R \cdot \frac{\mathrm{d} \phi}{\mathrm{d} t}$.
}
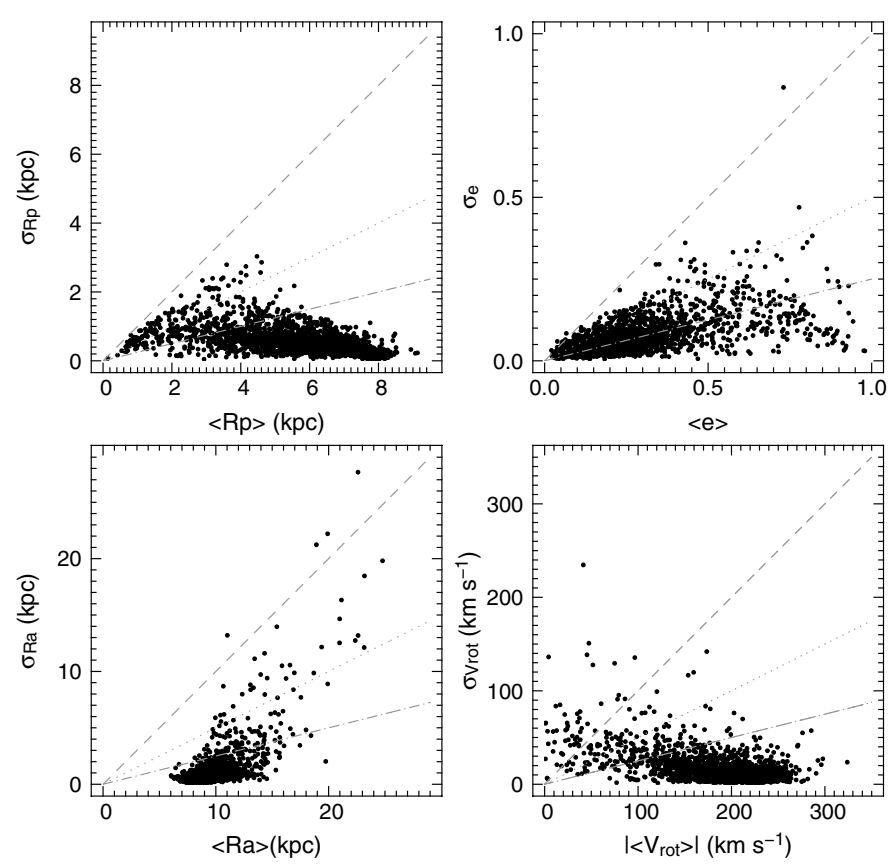

Fig. 3. Estimated uncertainties versus their respective stellar orbital parameters value for the SN75 sample. Dashed, dotted, and dash-dotted lines represent $100 \%, 50 \%$, and $25 \%$ errors, respectively.

(up to $3 \mathrm{kpc}$, see Figs. 2 and 5 top-right panel) for which the proper motions (and therefore the tangential velocities) are very uncertain, the orbital parameters show reasonably small variations when errors are taken into account. As showed in Fig. 3, the errors in eccentricity are smaller than 0.2 for most stars, while for $R_{\mathrm{p}}$ they are smaller than $1 \mathrm{kpc}$. Similar behaviour is seen in the other parameters. With such errors we are confident that we can use these orbital parameters to distinguish between thin- and thick-disc stars, with only moderate contamination.

During the preparation of this manuscript we used four different versions of distance estimations (two preliminary and one definitive, following the methods of Zwitter et al. 2010; and 
Binney et al., in prep.) and two different chemical catalogue versions (DR3 by Siebert et al. 2011; and DR4 by Kordopatis et al., in prep.) for our analysis. The results of the present work (see next sections) hold for all the data set we employed, confirming the robustness of the selected sample and the results obtained.

\section{The kinematical criteria of G03 applied to the RAVE data}

In G03 the authors used 150 field subdwarfs and early subgiants with accurate parallaxes and kinematics and divided them into three subsamples according to pure kinematic criteria. With the purpose of validating the RAVE abundance pipeline, as well as our orbital parameters, we follow here the analysis done in G03 and divide the RAVE samples into three subsamples according to the following criteria.

1. The thin disc component including stars whose orbits have low eccentricity and low maximum altitude from the Galactic plane, i.e. $e<0.25$ and $Z_{\max }<0.8 \mathrm{kpc}$. It will be represented as grey crosses in the figures that follow.

2. The dissipative collapse component consisting of stars with $V_{\text {rot }}>40 \mathrm{~km} \mathrm{~s}^{-1}$. Stars belonging to the thin disc component just defined are excluded. This sample includes part of the thick disc, as well as of the halo. Through all the figures this subsample will be represented with black dots.

3. The accretion component of non-rotating or counter-rotating stars. These stars satisfy the constraint $V_{\text {rot }} \leq 40 \mathrm{~km} \mathrm{~s}^{-1}$. This population is very likely composed of both halo stars and accreted debris (i.e. stars that do not share the rotation of the disc components). It is represented in the figures as open squares.

The criteria mentioned above deviate somewhat from the criteria used in G03, who defined the thin disc component via $\sqrt{Z_{\max }^{2}+4 e^{2}}<0.35$ and had an additional constraint on the apogalactic radius $R_{\mathrm{a}}<15 \mathrm{kpc}$ for the dissipative component. The thin disc stars selected by this constraint cannot have $e>0.175$ if they lie on the Galactic plane, and they must have circular orbits if their $Z_{\max }=0.35 \mathrm{kpc}$. Indeed, while the above criteria led to differences in the chemical composition that likely reflect real differences in the stellar populations (as will be shown in the next sections), the detailed specification is to some extent arbitrary, as already pointed out by G03. Our modification allows us to generalize the selection criteria in the $e-Z_{\max }$ plane, as we show in the next section. Furthermore, our sample covers a considerably larger volume than the HIPPARCos sphere probed by $\mathrm{G} 03$, resulting in a relatively low number of stars with near-circular and coplanar orbits when the G03 original selection criteria is employed.

Indeed, the G03 sample is composed of dwarf stars, covering a small spatial volume ( $<100 \mathrm{pc}$ from the Sun) in order to include only stars with accurate parallaxes. Moreover, their sample was drawn from the HIPPARCOS catalogue for which metal-poor stars were primarily selected (with $[\mathrm{Fe} / \mathrm{H}]<-0.8 \mathrm{dex}$ ). This sample therefore includes a rather large number of high-proper-motion stars, resulting in a strong kinematical bias favouring objects on highly eccentric orbits, with low Galactic rotation velocities, and with either large apogalactic or small perigalactic distances.

The RAVE sample considered here, on the other hand, is composed of giant stars, thus, probing a large volume of space (up to $3 \mathrm{kpc}$ from the Sun). Most importantly, the RAVE sample is rich in high metallicity $(>-1.0 \mathrm{dex})$ stars. Moreover, while

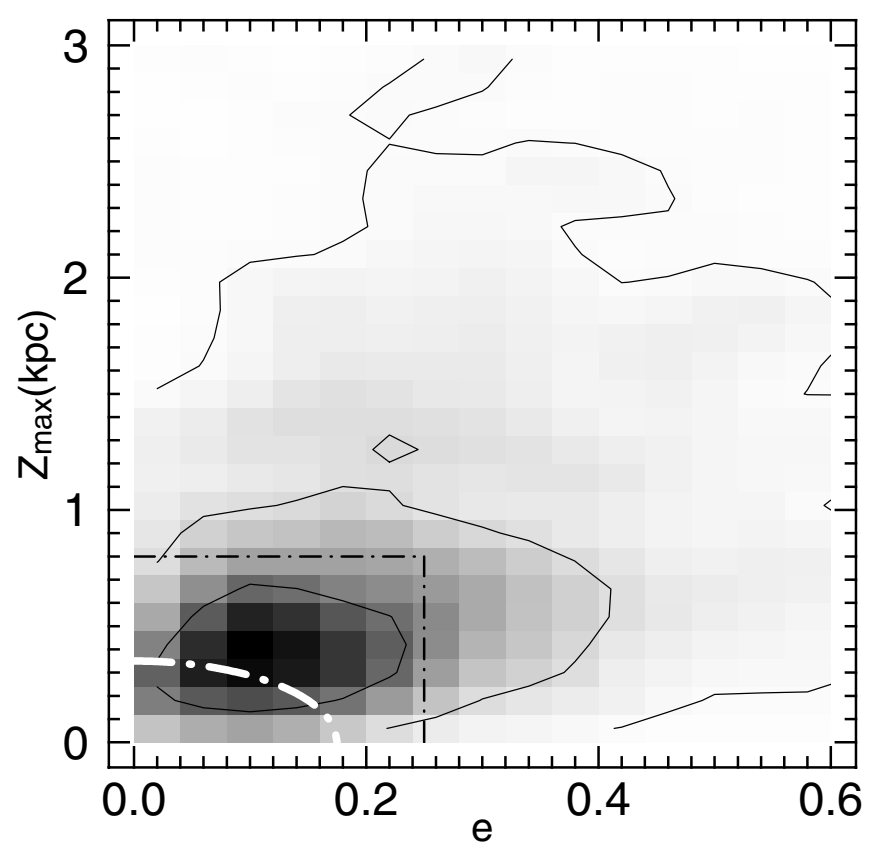

Fig. 4. Density distribution on the $e-Z_{\max }$ plane for our sample SN75 sample, with 2167 stars. The dash-dotted white curve shows the G03 thin-disc selection criteria, whereas the dashed-dotted black line frames the area defined by our modification to the $e$ and $Z_{\max }$ constraints. The contours contain $34 \%, 68 \%$ and $95 \%$ of the sample.

the G03 sample is composed of different high-resolution spectroscopic subsamples, partly obtained by the authors and partly found in the literature, the RAVE sample adopted here is very homogeneous. Finally, our samples SN75 and SN60 respectively have $\sim 14$ and $\sim 60$ times the size of the G03 sample.

In Fig. 4 we show number density contours of the maximum height achieved above the Galactic plane $Z_{\max }$ versus the orbital eccentricities $e$ for the sample SN75. The criterion $\sqrt{Z_{\max }^{2}+4 e^{2}}<0.35$ adopted by G03 to define the thin-disc sample is represented by the quarter of an ellipse on the $e-Z_{\max }$ plane and covers only part of the the thin disc defined by our criterion.

With the above prescriptions, the thin-disc component consists of 1079 stars, the dissipative component of 1024 stars, and the accretion component of 64 stars. The results of this division are shown in Figs. 1, 2, 5-8. The thin disc, dissipative, and accretion components show different chemical signatures, similar to the ones seen in G03, with the difference that our sample extends up to solar abundances.

Figure 5 shows the main properties of the different samples. While the accretion component appears scattered over a wide area of the Toomre diagram (and it would extend to higher $v$, since such non-rotating population must hold rotating and counter-rotating stars in equal amounts) the thin disc and dissipative components clump and overlap on a smaller area. With respect to the distances, the outcome of our sample selection is that the thin disc stars cover a smaller volume than the dissipative sample, whereas the accreted stars prefer larger distances (between 0 and $3 \mathrm{kpc}$ ) from the Sun. The eccentricity distribution of each sample is also shown. It is interesting to notice that the eccentricity and distance distributions found here are qualitatively similar to those found with the SEGUE G-dwarf sample used in Lee et al. (2011), even though these authors adopted a pure chemical criterion to divide their sample 
A\&A 553, A19 (2013)
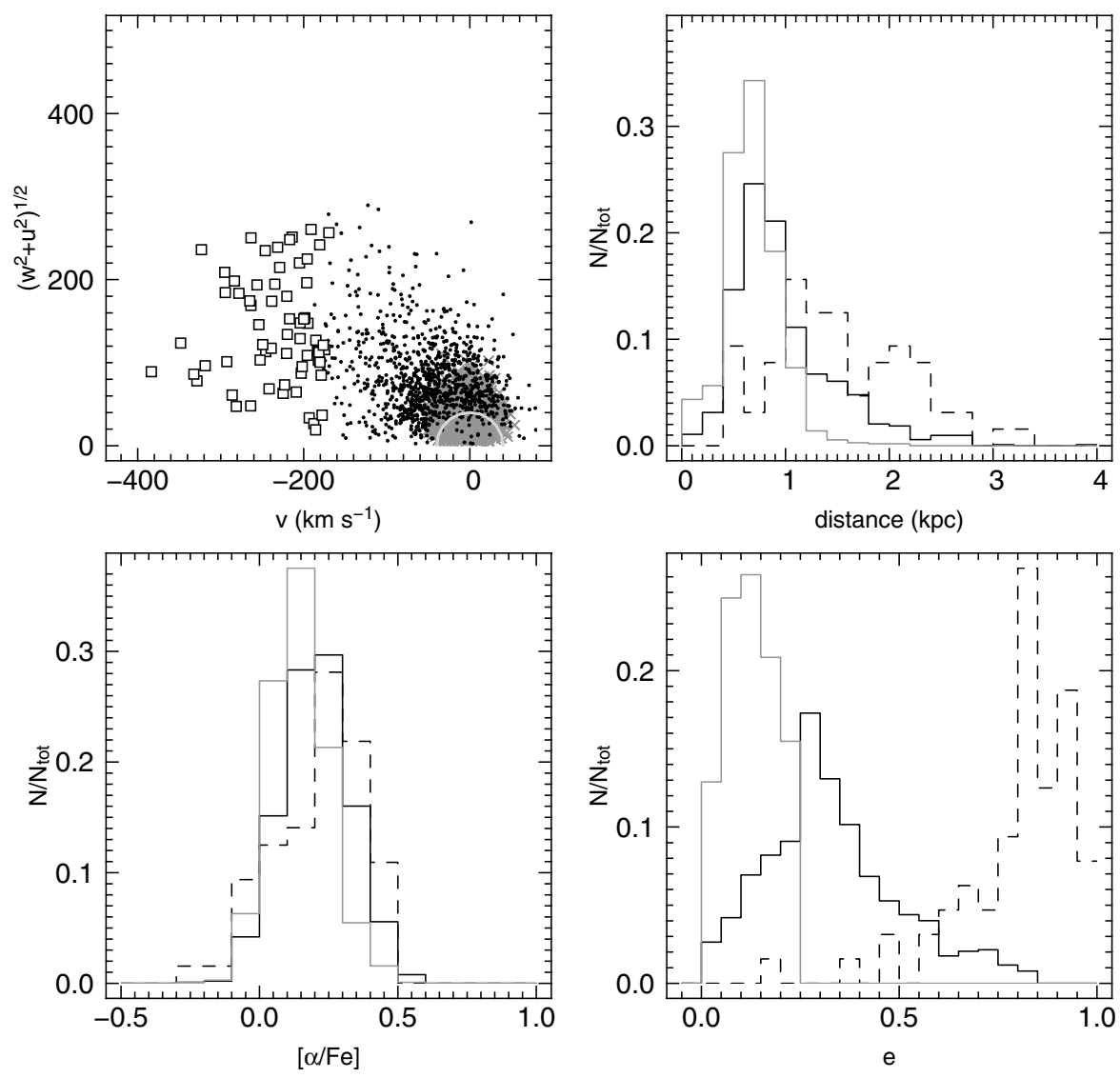

Fig. 5. Toomre diagram (upper left) for each of our samples: thin disc stars (grey points), dissipative component (mostly thick disc stars - black dots), and accretion component (squares). The light grey semi-circle indicates the constant peculiar velocity $\left(u^{2}+v^{2}+w^{2}\right)^{1 / 2}=$ $40 \mathrm{~km} \mathrm{~s}^{-1}$. Also shown are the distance (upper right), $[\alpha / \mathrm{Fe}]$ (bottom left), and eccentricity (bottom right) distributions of the thin disc (grey line) dissipative (solid black line) and accretion (black dashed line) components.
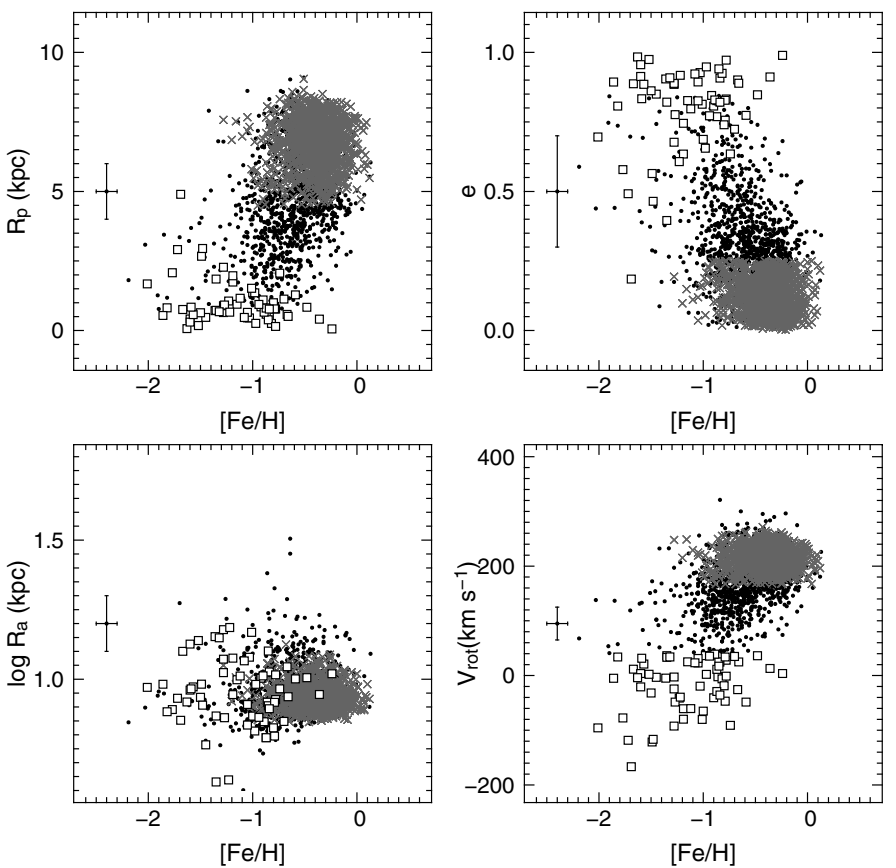

Fig. 6. Perigalacticon, $R_{\mathrm{p}}$, eccentricity, $e$, apogalacticon, $R_{\mathrm{a}}$, and Galactic rotation velocity, $V_{\text {rot }}$ as a function of $[\mathrm{Fe} / \mathrm{H}]$ for the three subsamples. Symbols are as in Fig. 5. This figure corresponds to Fig. 4 of G03.

into thin- and thick-disc stars and even though our distance and eccentricity distribution is likely to be affected by our selection criteria.
Indeed, the RAVE giant sample covers essentially the same volume as the SEGUE dwarf sample (Steinmetz 2012). The similarities between our thin and dissipative samples and the thin and thick disc ones obtained by Lee et al. (2011), is by itself reassuring. Indeed, two completely different surveys, using different tracers (giants vs. dwarfs), with chemistry, distances, and orbital parameters computed by different pipelines, analysed in completely different ways (kinematically selected vs. chemically selected), still give very similar answers for the mix of Galactic stellar populations within $\sim 2-3 \mathrm{kpc}$ from the Sun.

In Fig. 5 we also show the $[\alpha / \mathrm{Fe}]$ distributions for each of our samples. An offset of $\sim 0.1$ dex is seen between the thin and dissipative samples, however with considerable overlap (as expected even according to pure chemical evolution models, where the difference in $[\alpha / \mathrm{Fe}]$ between the thick and thin discs is a function of metallicity - see Chiappini 2009). The absolute values of the mean $[\alpha / \mathrm{Fe}]$ ratios of the thin disc and thick disc (dissipative) distributions are in good agreement with the mean values reported by high-resolution studies (Bensby \& Feltzing 2012, and references therein). These values are, however, lower than the mean values obtained by Lee et al. (2011) with the SEGUE sample, namely: $[\alpha / \mathrm{Fe}] \sim 0.1$ for the thin disc, and $\sim 0.35$ for the thick disc (a detailed comparison between RAVE and SEGUE results is beyond the scope of the present paper, and will be presented by Brauer et al., in prep.).

In Figs. 6-8 we successfully reproduced G03's Figs. 4-6, respectively, with some additional features. The RAVE data support some of the G03 results, such as the existence of correlations between $[\mathrm{Fe} / \mathrm{H}]$ and $R_{\mathrm{p}}, e, V_{\text {rot }}$ (see also Spagna et al. 2010), and the apparent absence of a correlation with $R_{\mathrm{a}}$ (Fig. 6). The dissipative component has a wide range of eccentricity and moderately high abundances. Also seen in Figs. 6 and 7 is an apparent drop in the black point density around $[\mathrm{Fe} / \mathrm{H}] \sim-1.0 \mathrm{dex}$ 
C. Boeche et al.: The relation between chemical abundances and kinematics of the Galactic disc with RAVE
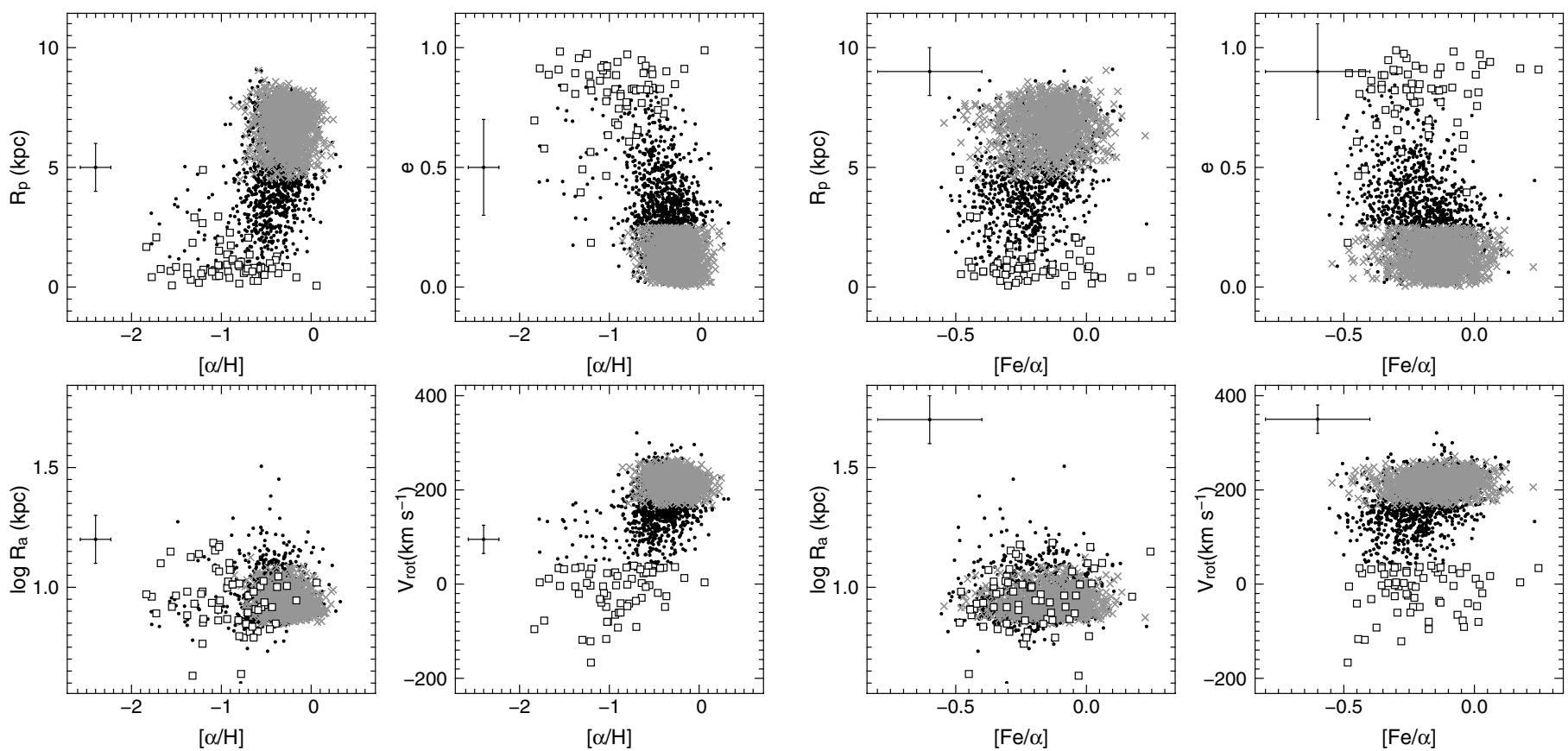

Fig. 7. As in Fig. 6. This figure corresponds to Fig. 5 of G03.

and $[\alpha / \mathrm{H}] \sim-0.7$ dex (see also Figs. 9 and 10 top and middle panels). The standard deviation of the black points is $\sigma_{[\alpha / \mathrm{H}]}=$ 0.2 dex (after the rejection of the accretion component stars polluting the dissipative component at $[\alpha / \mathrm{H}]<-1.0)$, which is very close to the error in abundance expected for $[\alpha / \mathrm{H}](0.17 \mathrm{dex})$. This supports the weak correlation shown with $V_{\text {rot }}$ and -0.7 dex may represent the lower limit of the thick disc $\alpha$-abundance.

In Figs. 9 and 10 we show the trends in $[\mathrm{Fe} / \alpha]$ with $[\alpha / \mathrm{H}]$, and $[\alpha / \mathrm{Fe}]$ with $[\mathrm{Fe} / \mathrm{H}]$ (similar to Figs. 2 and 3 of $\mathrm{G} 03$, respectively).

Both the thin and dissipative components show a decreasing $[\alpha / \mathrm{Fe}]$ abundance ratio with increasing metallicity. However, the thin-disc component shows systematically higher $[\mathrm{Fe} / \alpha]$ ratios than the dissipative one for $[\alpha / \mathrm{H}]<-0.2$ dex. By plotting a fiducial line in each panel, the difference between the thin-disc and dissipative component is visible in Fig. 9. For $[\alpha / \mathrm{H}]>-0.2$ dex the thin and dissipative components seem to be chemically indistinguishable. For the accreted component the abundance ratio essentially remains flat, and more importantly, shows systematically lower $[\alpha / \mathrm{Fe}]$ than the dissipative component (see bottom panel of Fig. 10). This result not only agrees with the original findings of G03, but is also similar to the recent high-resolution results by Nissen $\&$ Schuster (2010).

It is clear that although the G03 criteria lead to clear subpopulations, overlaps between the samples still exist. The $\alpha$-enhanced stars at $[\mathrm{Fe} / \mathrm{H}]<-0.7$ dex of the thin-disc component (Fig. 10, top panel) probably belong to the dissipative component. On the other hand, the stars at $[\mathrm{Fe} / \mathrm{H}]<-1.0$ dex of the dissipative component are very likely accretion component stars. In fact, because the accretion component stars are nonrotating (they can be identified as halo stars by looking at the locus they occupy in the Toomre diagram, top-left panel of 5), their average $V_{\text {rot }}$ must be zero. By mirroring the square points with respect to $V_{\text {rot }}=0\left(v=-217 \mathrm{~km} \mathrm{~s}^{-1}\right)$, we can infer that many black points (dissipative component) could also be considered accretion component stars.
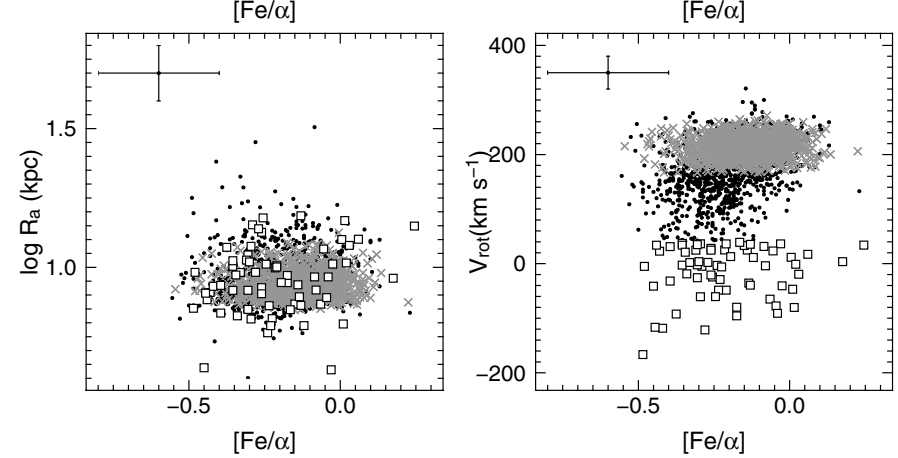

Fig. 8. As in Fig. 6. This figure corresponds to Fig. 6 of G03.

Despite the small overlaps described above, the three groups of stars assigned to the different components via kinematical criteria show different chemistry:

- thin disc component: the distributions in $R_{\mathrm{a}}, R_{\mathrm{p}}, e$, and $V_{\text {rot }}$ span a narrow range of values because they are limited by the criteria $e<0.25$ (nearly circular orbits) and $Z_{\max }<0.8 \mathrm{kpc}$ (close to the Galactic plane); i.e., stars are contained in a local volume with $R=8 \pm 1 \mathrm{kpc}$, see Fig. 2 . This component has an $[\alpha / \mathrm{Fe}]$ peak slightly above zero and tends to be more shifted to higher metallicities (Figs. 6 and 7).

- dissipative component: for this subsample $V_{\text {rot }}$ is limited downward by the constraint $V_{\text {rot }}>40 \mathrm{~km} \mathrm{~s}^{-1}$ and stars that happen to have circular orbits $(e<0.25)$ are missing from this component in favour of the thin disc component. This creates a small bias against the dissipative component that diminishes the number of stars at low eccentricities, but does not completely remove them, since the orbital parameters can still span a wide range of values for $R_{\mathrm{p}}$ and $e$ as well. On the other hand, stars at $[\mathrm{Fe} / \mathrm{H}]<-1.0$ dex are likely to be members of the accretion component. The dissipative component has more stars at high $[\alpha / \mathrm{Fe}]$ with respect to the thin-disc component. It also shows a correlation between the abundances $[\mathrm{Fe} / \mathrm{H}],[\alpha / \mathrm{H}]$ and the orbital parameters, as also found by $\mathrm{G} 03$.

- accretion component: the kinematical criteria adopted for this sample imply that such objects have mainly high eccentricity (Fig. 5). This component covers a wide range in $[\mathrm{Fe} / \mathrm{H}]$, including objects with clearly higher metallicities than the upper limit halo stars metallicity of $\sim-1$.

The successful reproduction of the main results of G03 validates the kinematical and chemical data of the RAVE survey (even though they were obtained from medium-resolution spectroscopy) and allows us to push our analysis further.

However, any selection criteria aiming at disentangling the thin and thick discs will suffer from the fact that these two components overlap in almost all parameters. Pure kinematical selection criteria of thin and thick disc return samples which 

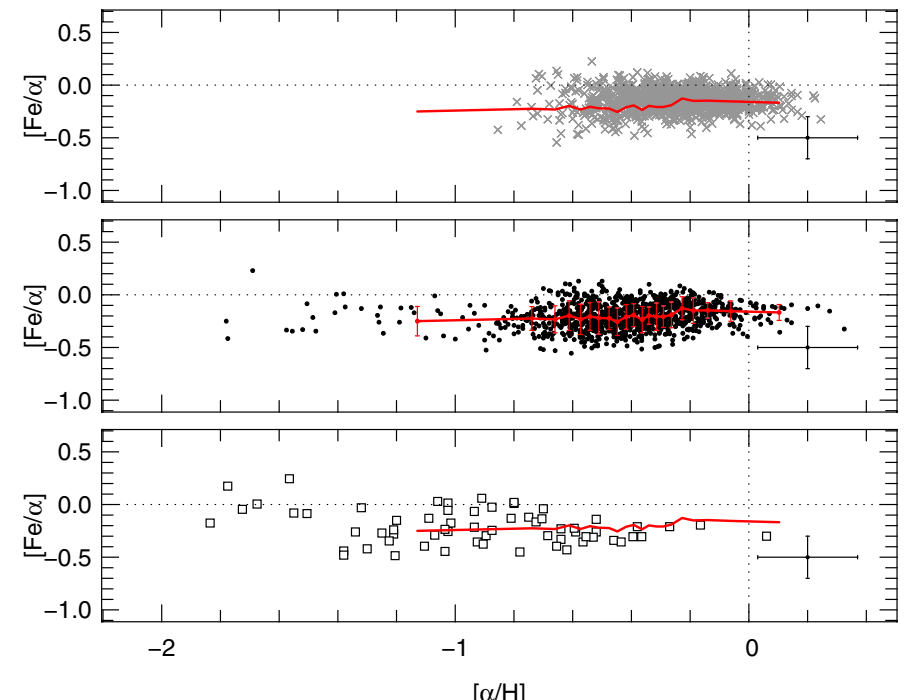

$[\alpha / \mathrm{H}]$

Fig. 9. Abundance ratio $[\mathrm{Fe} / \alpha]$ versus the abundance $[\alpha / \mathrm{H}]$ for the thin disc component (grey crosses, top panel), the dissipative component (black points, middle panel), and the accretion component (open squares, bottom panel), selected by using our modified criteria (see text). The red line represents the average $[\mathrm{Fe} / \alpha]$ of the dissipative component obtained by averaging bins of 50 points each, and the error bars represent their standard deviation. The red line is reproduced in each panel as a fiducial line. This figure corresponds to Fig. 2 of G03.

partially overlap in chemical abundances (Bensby et al. 2003, 2005; Reddy et al. 2006, among others), whereas pure chemical selection criteria return samples that partially overlap in kinematics (Navarro et al. 2011; Lee et al. 2011). Even if a clear kinematical separation between these two components did exist in the past, it could have been heavily blurred by a number of agents. In the next section we try an alternative approach with the aim of avoiding strict selection criteria, which hopefully can be more successful in providing more robust constraints on chemodynamical models.

\section{The $e-Z_{\max }$ plane}

As discussed in Sect. 1, there are two general processes that result from the disc secular evolution, which could partially destroy the kinematical borders between the thin and thick discs: (1) kinematical heating (increase in stellar velocity dispersion) with time and (2) radial migration (change in the angular momentum of stars and, thus, in their guiding radii). Another possibility is (3) the deposition of material by accretion, making the situation even more complex since mergers also can give rise to (1) and (2) above. Processes (1), (2), and (3) will lead to different signatures:

(1) Heating by transient (Carlberg \& Sellwood 1985) and multiple (Minchev \& Quillen 2006) spiral density waves, the Galactic bar (Minchev \& Famaey 2010), giant molecular clouds (Jenkins \& Binney 1990), and minor merger events (Quinn et al. 1993) would most probably increase ${ }^{6}$ the velocity dispersions of all three components $(U, V$, and $W)$. An increase in the vertical velocity dispersion would then result in overlapping an initially cooler stellar population (such as the thin disc), for which the heating is more effective due to

\footnotetext{
6 However, this effect still needs to be better quantified for an MW-like galaxy.
}

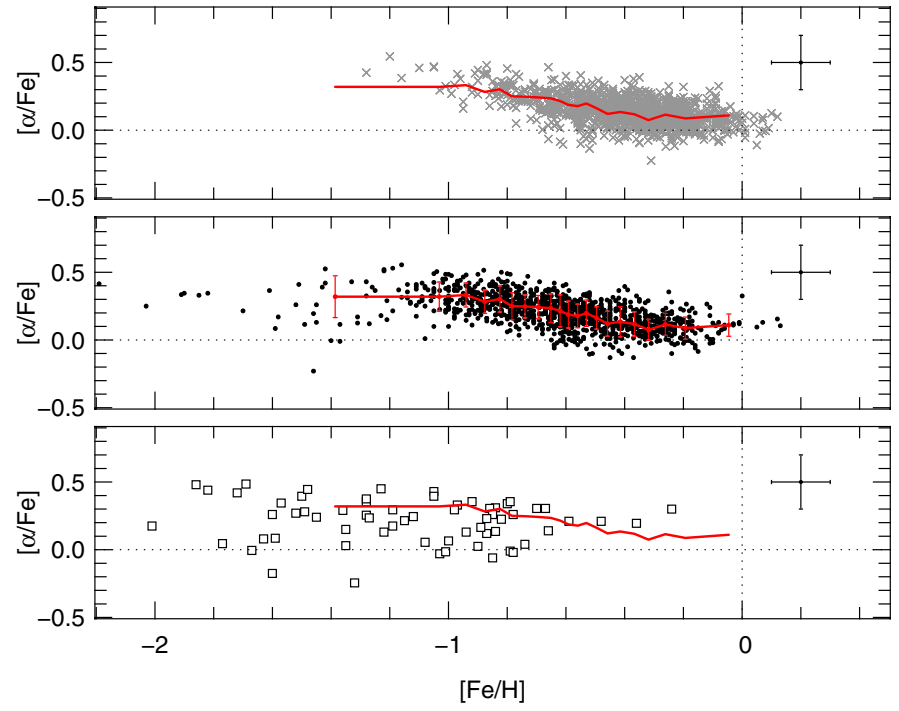

Fig. 10. As in Fig. 9 but for the abundance ratio $[\alpha / \mathrm{Fe}]$ now as function of $[\mathrm{Fe} / \mathrm{H}]$. This figure corresponds to Fig. 3 in G03.

the cold orbits, with an initially hotter stellar sample (such as an old thick disc), which would not be affected much by the heating agents. The above would be true irrespective of whether the thick disc were born hot (Forbes et al. 2012) or were preheated by mergers at high redshift (e.g., Villalobos $\&$ Helmi 2008) by virtue of the thin disc being younger than the thick disc and the expected decrease in merger activity with redshift.

(2) In contrast, as a star migrates (gains or loses angular momentum), information about its birth radius is lost, and its kinematics can be virtually indistinguishable from those of stars born at the new radius (Sellwood \& Binney 2002; Minchev et al. 2012a, 2012c). In recent years radial migration (or mixing) has been recognized as an important process affecting galactic discs. Several radial migration mechanisms have been described in the literature: the effect of the corotation resonance of transient spiral density waves (Sellwood \& Binney 2002; Roškar et al. 2008; Schönrich \& Binney 2009a), the effect of the non-linear coupling between multiple spiral waves (Minchev \& Quillen 2006) or bar and spirals (Minchev et al. 2010, 2011; Brunetti et al. 2011), and the effect of minor mergers (Quillen et al. 2009; Bird et al. 2011).

(3) Deposition of material by accretion has been shown to commonly occur in cosmological simulations (e.g., Abadi et al. 2003). In this scenario tidal debris of satellites with orbits coinciding with the plane of the host disc can populate a disc component and, thus, blur the borders between the preexisting thin and thick discs.

Such diffusion mechanisms make any kinematic criteria aiming at separating the thin from the thick discs ineffective, even in the unrealistic case of no errors in the kinematical data available. The chemical properties acquired at birth, on the other hand, must be preserved. However, here the difficulty is that due to the shallow abundance gradient in the galactic disc, as well as the narrow range in the $[\alpha / \mathrm{Fe}]$ variation among different galactic populations (at most $0.5 \mathrm{dex}$ ), the chemical differences are subtle (unless one uses abundance ratios involving other chemical elements that present larger variations). In addition, even if the differences in the star formation histories of the thick and 


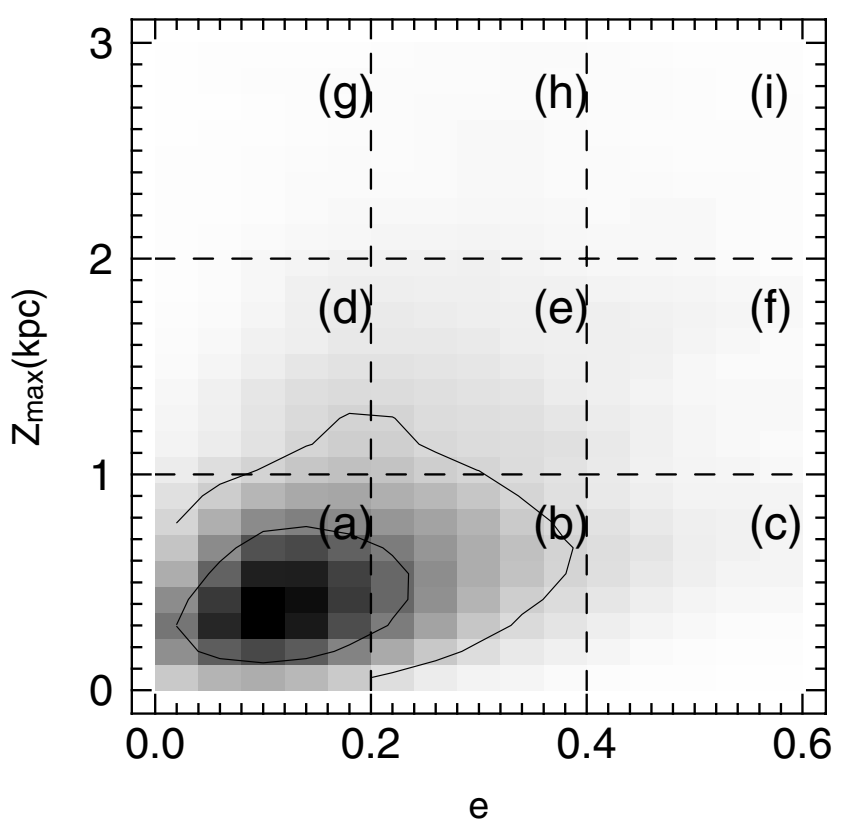

Fig. 11. $e-Z_{\max }$ plane divided in nine panels labelled from a) to i). Here we use the SN60 sample, which counts 9131 stars.

thin discs led to chemical differences (see Chiappini 2009), large overlaps would still exist. This would remain true if the disc was composed of several mono-abundance subcomponents, as suggested by Bovy et al. (2012). For these reasons, we abandon the "selection criteria" approach and try to take a different view of the problem.

Our previous analysis considered the distributions of the orbital parameters (such as $Z_{\max }, R_{\mathrm{p}}, e$, and $V_{\text {rot }}$ ) separately. However, these distributions are slices in a bigger chemokinematical, multidimensional space, in which the stars lie. It is therefore possible that some information could be missed when only the shapes of these distributions are considered, resulting in a mixup in our separation of different Galactic components. For instance, consider two stars with $V_{\text {rot }} \sim 220 \mathrm{~km} \mathrm{~s}^{-1}$ : one can have a circular orbit on the Galactic plane at the Sun radius $R_{0}$, and the other can have $R_{\mathrm{p}}<R_{0}$, an eccentric orbit and vertical velocity $v_{z}$ high enough to reach $Z_{\max }>2 \mathrm{kpc}$. It could hardly be thought that such stars belong to the same population. To avoid such a trap, we analyse further our sample by grouping the stars by similar orbits using the $e-Z_{\max }$ plane. The eccentricity gives the shape of the orbits, whereas $Z_{\max }$ tells us about the oscillation of the star perpendicular to the Galactic plane.

In Fig. 11 we divide the $e-Z_{\max }$ plane in nine groups of stars, and we label them from (a) to (i). We have neglected stars with $e>0.6$ because here we focus on the Galactic disc. In this way we have sorted the stars into "classes" of orbits: moving rightwards the eccentricity grows, moving upwards $Z_{\max }$ grows (so the vertical $v_{z}$ velocity increases).

By dividing the $e-Z_{\max }$ plane into nine regions, we have obtained stellar subsamples with narrow ranges in orbital parameters. For each group we now plot the $[\alpha / \mathrm{Fe}]$ versus $[\mathrm{Fe} / \mathrm{H}]$ relation and the distributions of $[\mathrm{Fe} / \mathrm{H}],[\alpha / \mathrm{Fe}], R_{\mathrm{p}}, R_{\mathrm{m}}$, and $V_{\text {rot }}$, in Figs. 12 and 13, respectively. Panels (a), (c), and (g) of these figures give particularly interesting insights, so we now focus on them.

\subsection{Identification of thin/thick/diffused stars}

In the following analysis we use the SN60 sample, in order to have a more robust statistic ${ }^{7}$. We first study the distributions of subsample (a), as defined in Fig. 11. The stars in this group (with eccentricities $<0.2$ and low vertical velocities) show the expected properties of a sample dominated by local thin-disc stars, namely: an Fe-distribution peak at $\sim-0.25$ dex, a $V_{\text {rot }}$ peak at $\sim 220 \mathrm{~km} \mathrm{~s}^{-1}$, and a mean galactocentric distance $R_{\mathrm{m}}$ of $\sim 7.5 \mathrm{kpc}$. The apparent low abundance of the Fe-distribution (which peaks at $\sim-0.25$ dex instead of $\sim-0.05$ dex of the local thin disc found by Casagrande et al. 2011) is due to the spatial distribution of the RAVE sample, which lacks nearby stars and favours stars lying at $z_{\mathrm{Gal}}>300 \mathrm{pc}$, where the metallicity distribution function is shifted to lower values (also found by Schlesinger et al. 2012, and is consistent with the predictions of the chemodynamical model of Minchev et al. 2012d). Nonetheless, the Fe-distribution in panel (c) is more metal rich in comparison to the subsamples of the other panels.

By moving up from panel (a) to panel ( $\mathrm{g}$ ) the mean $R_{\mathrm{p}}$ and $R_{\mathrm{m}}$ do not change significantly but their distributions become broader (see Fig. 13 top panel), whereas the $V_{\text {rot }}$ distribution decreases its mean slightly from $220 \mathrm{~km} \mathrm{~s}^{-1}$ to $200 \mathrm{~km} \mathrm{~s}^{-1}$ (see Fig. 12 bottom panel). The Fe-distribution is found to shift to lower abundances (with a peak around -0.6 dex, Fig. 12 top panel), and the average $[\alpha / \mathrm{Fe}]$ increases by $\sim 0.1$ dex. All the above properties are indicative of a sample dominated by local thick disc stars (panel g). However, the presence of kinematically heated old thin disc stars cannot be discarded. Stars with high $Z_{\max }$ and low eccentricities must be on orbits that oscillate strongly through the Galactic plane, suggesting that they have experienced some perturbations during their lifetimes. Feltzing \& Bensby (2008) propose the same interpretation for a subsample of 32 stars that would lie in panel (d) of our Fig. 11. The cause for this might be identified in the kinematical heating mechanism proposed by some authors (e.g. Villalobos \& Helmi 2008; Bournaud et al. 2009; Minchev et al. 2012d, for different processes) in order to explain the thickness of the disc.

Focusing now on panel (c) of Figs. 12 and 13 (upper panels), we identify a population with high eccentricities but confined close to the disc plane $\left(Z_{\max }<1 \mathrm{kpc}, 0.4>e>0.6\right)$. Stars in this group have small perigalactic radii $\left(R_{\mathrm{p}} \sim 3 \mathrm{kpc}\right)$ and small mean radii $\left(R_{\mathrm{m}} \sim 6 \mathrm{kpc}\right)$. Due to their high eccentricities and the large fraction of stars coming from the inner disc, the observed $V_{\text {rot }}$ distribution (Fig. 13, lower panel) peaks at $\sim 120 \mathrm{~km} \mathrm{~s}^{-1}$, and has a tail extending up to more than $200 \mathrm{~km} \mathrm{~s}^{-1}$. In addition, stars in this panel show a broad Fe-distribution (Fig. 12, upper panel) with a hint of bimodality. The latter differs significantly from the metallicity distributions in panels (a) or (b) (a Kolmogorov-Smirnov test showed that the probability that the distribution in panel (c) is drawn from the populations of panel (a) or (b) is both lower than $10^{-17}$ ). We note also that, by moving upward from panel (c) to panel (i), the high $[\mathrm{Fe} / \mathrm{H}]$ tail of the distribution progressively disappears, leaving an Fe-distribution qualitatively similar to the thick disc one. This means that in panel (c) there are most probably more than one population, i.e. stars that might belong to both the thin and thick discs. While stars with low $\mathrm{Fe}$ abundance $([\mathrm{Fe} / \mathrm{H}]<-0.5$ dex $)$ have all the characteristics needed to be identified as thick disc, stars with high $\mathrm{Fe}$ abundance $([\mathrm{Fe} / \mathrm{H}]>-0.4 \mathrm{dex})$ are likely to be thindisc stars scattered outward from the inner part of the Galaxy. Because such stars are drawn from panel (c), their kinematic

7 We verified that the results found in this work are valid and consistent for both SN75 and SN60 samples. 

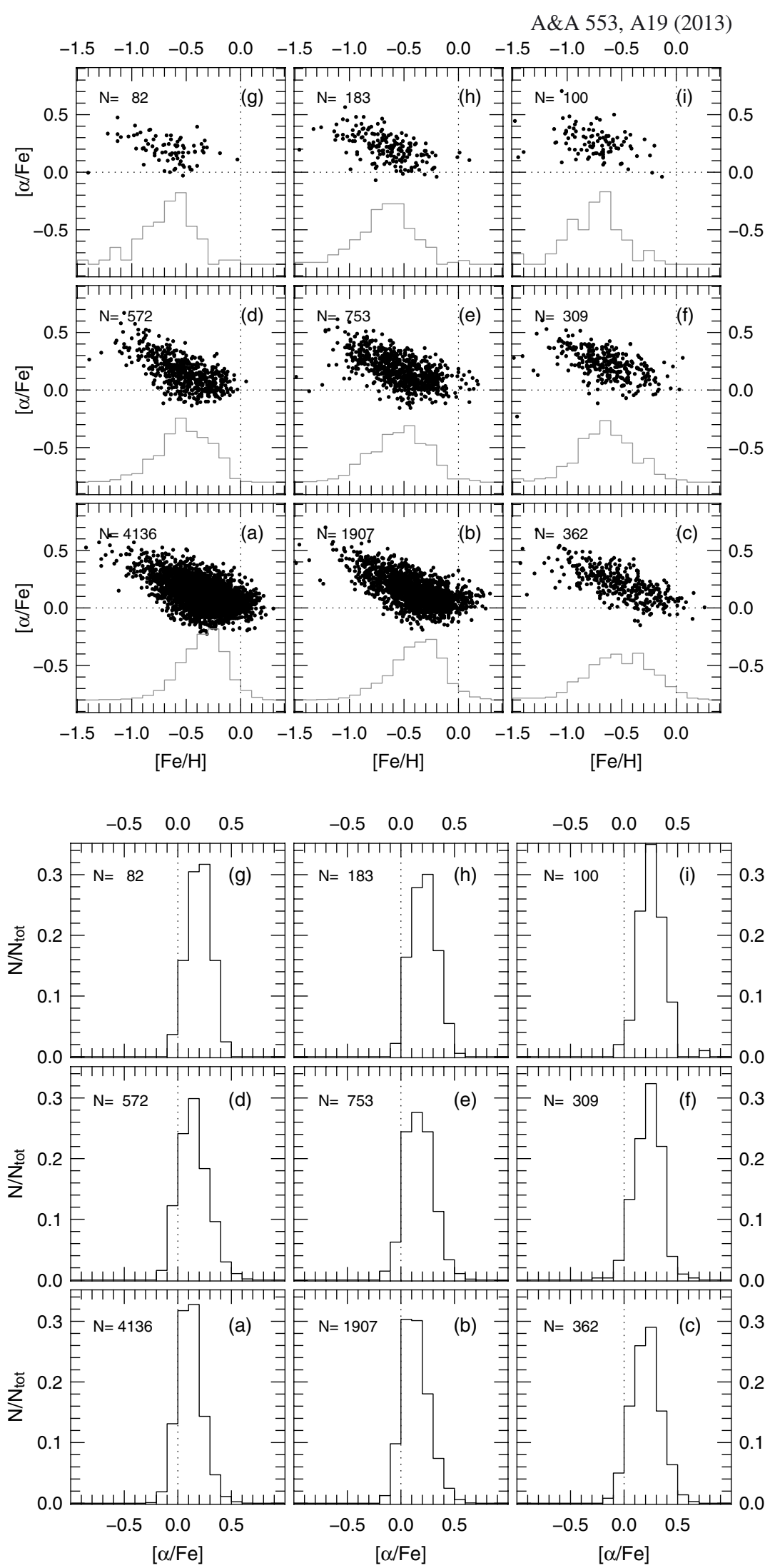

Fig. 12. Upper panel: relative abundance $[\alpha / \mathrm{Fe}]$ versus $[\mathrm{Fe} / \mathrm{H}]$ for the stellar samples defined by panels a) through i) in Fig. 11. The histograms represent the Fe distributions with relative scales. Lower panel: distributions of abundance $[\alpha / \mathrm{Fe}]$ for the stellar samples defined by panels a) through i) in Fig. 11. The distributions are normalized over the total number of points contained in each panel $\left(N_{\text {tot }}\right)$.

is determined and thick-disc-like, but their chemical abundance is typical of the thin-disc. Therefore, they have no clear thinthick-disc classification. This feature is highlighted in Fig. 14 where the distributions in $V_{\mathrm{rot}}, R_{\mathrm{m}}$ and $[\alpha / \mathrm{Fe}]$ of the two tails are shown separately. Kinematically, the stars belonging to the two tails show no significant differences, whereas they have distinct chemical abundances in $[\mathrm{Fe} / \mathrm{H}]$ and $[\alpha / \mathrm{Fe}]$.

Such stars having thick-disc kinematics and thin-disc chemical abundances might have been kinematically heated and/or migrated by a mechanism that scatters stars out from the inner 
C. Boeche et al.: The relation between chemical abundances and kinematics of the Galactic disc with RAVE
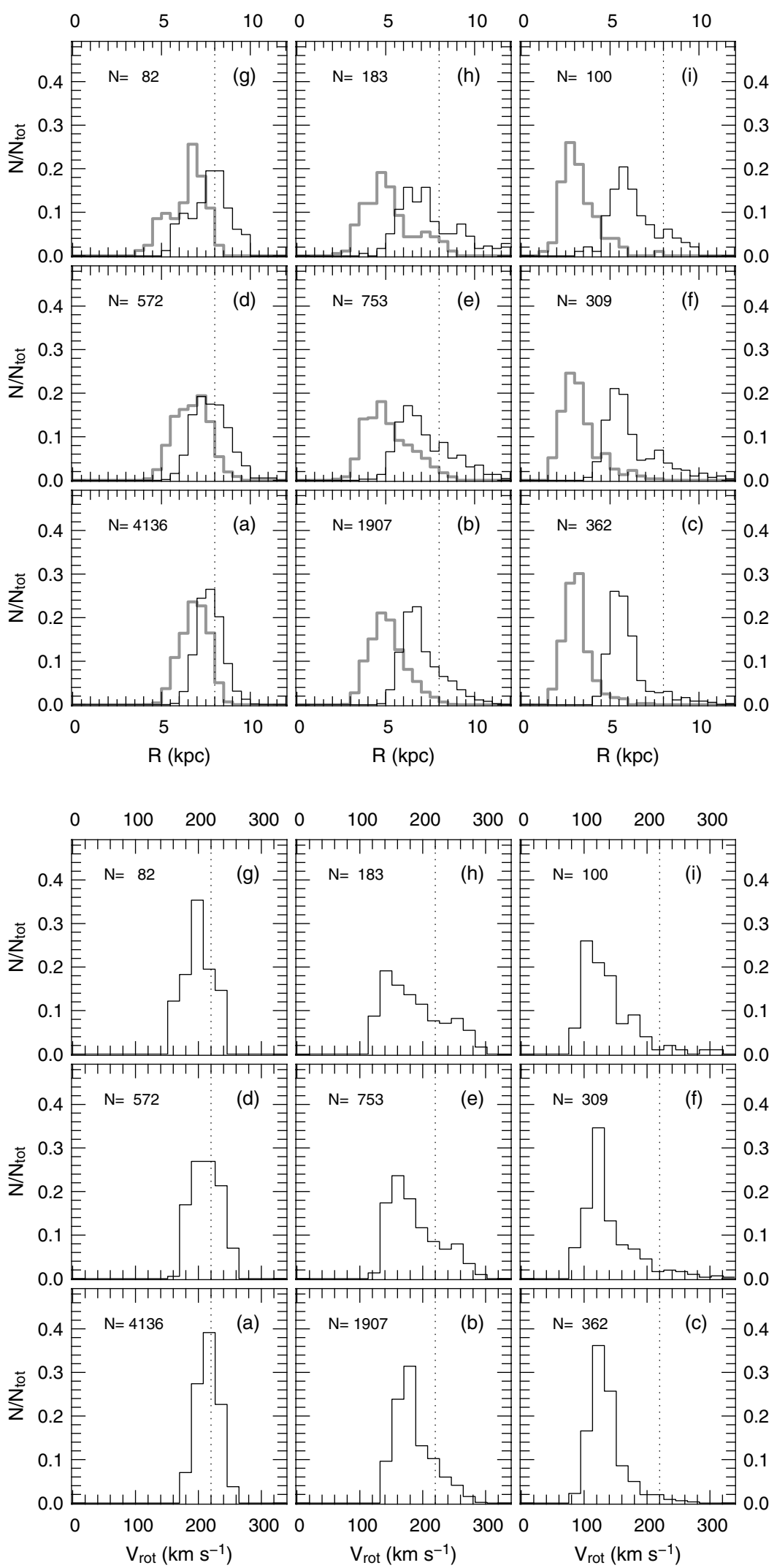

Fig. 13. Upper panel: perigalacticon (grey lines) and mean radius (black lines) distributions for the stellar samples defined by panels a) through i) in Fig. 11. The dotted line indicates $R=8 \mathrm{kpc}$. Lower panel: rotational velocities $V_{\text {rot }}$ for the stellar samples defined by panels a)-i) in Fig. 11. The dotted line indicates $V_{\text {rot }}=220 \mathrm{~km} \mathrm{~s}^{-1}$. parts of the Galaxy, and could be identified as the gravitational actions of the spiral arms (Schönrich \& Binney 2009a; Roškar et al. 2008) and/or the Galactic bar (Minchev \& Famaey 2010; Minchev et al. 2011; Brunetti et al. 2011).
The identification of two populations coming from the inner parts of the Galaxy, lying in the Galactic plane but having different metallicities, suggests that thick-disc and dynamically heated and/or migrated stars are separate populations with different 


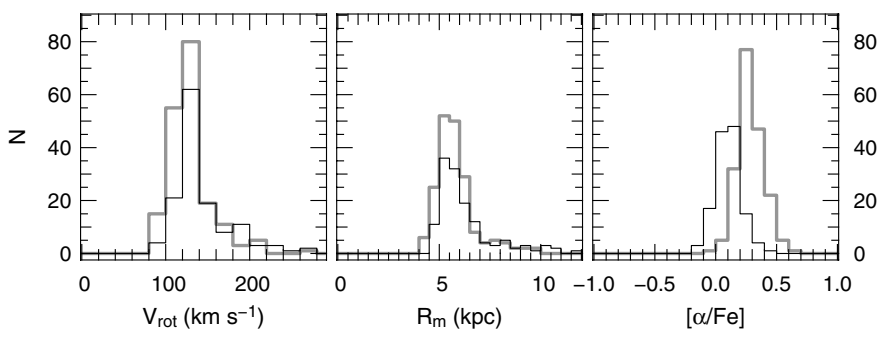

Fig. 14. Distributions in $V_{\text {rot }}$ and $R_{\mathrm{m}}$ of the high metallicity tail $([\mathrm{Fe} / \mathrm{H}] \geq-0.4$ dex, black line) and low-metallicity tail $([\mathrm{Fe} / \mathrm{H}]<$ -0.5 dex, grey line) of the distribution in Fig. 12 top, panel c).

origins and evolutions. This observation is consistent with the previous findings by (Wilson et al. 2011; Liu \& van de Ven 2012; Kordopatis et al. 2011b), based on the eccentricity distribution of thick disc stars and challenges scenarios in which the thick disc formed solely by the outward migration of stars born in the inner disc (Schönrich \& Binney 2009a), while on the other hand supports the predicted existence and action of such a mechanism (see also Grenon 1999; Trevisan et al. 2011). Our conclusion also agrees with the recent work by Minchev et al. (2012d), who suggest that both mergers at early phases and the effect of a central bar at later times are needed to explain the presence of stars with thick-disc chemistry and kinematics currently found in the solar vicinity.

That we find such stars in panel (c) does not exclude the possibility that heated or migrated stars might be found in other regions of the $e-Z_{\max }$ plane. For instance, as mentioned in Sect. 5, migrated stars can move away from their original Galactic radius and conserve their eccentricity, making them indistinguishable from the locally born stars. In such case only the chemical composition can reveal the difference between migrated and local stars, but in our sample, the identification of such stars is challenging, because to draw a clear chemical signature requires a number of measured elements and a higher precision in abundance than the ones provided by RAVE.

On the other hand, kinematically heated stars would have a high probability to get into high-eccentric orbits. In doing so, they would enrich the poorly populated tail of the eccentricity distribution of the local stars, and broaden the $[\mathrm{Fe} / \mathrm{H}]$ distribution, making it more likely to see a bimodality within the error. This makes panel (c) a more favourable region for seeking such stars.

\section{Discussion and conclusions}

We selected two samples of 2167 and 9131 giant stars from the RAVE chemical catalogue having good quality spectra $(S / N>$ 75 and $S / N>60$, respectively called "SN75" and "SN60" sample), and known kinematics. We applied the kinematic criteria by G03 to the SN75 sample aiming at disentangling different populations of the Milky Way. Following G03, we divided the SN75 sample into thin disc, dissipative component, and accretion component. We verified first that our data (obtained from medium-resolution spectra) matches the results by G03 obtained from high-resolution spectra well, thereby confirming the reliability of the RAVE kinematic and chemical abundances.

Our thin disc and dissipative (mostly thick disc) samples selected by pure kinematical criteria turned out to have similar distance and eccentricity distributions to those reported by Lee et al. (2011) for a G-dwarf SEGUE sample where the thin and thick disc stars were selected based on a purely chemical criterion.
We also notice that thin-disc, dissipation, and accretion components partially overlap in several parameters. Such overlaps are to be expected if the processes currently debated in the literature (accretion, heating, and stellar migration) are at play during the evolution of the Milky Way. This is particularly true for thin and thick discs (the latter is identified with the dissipation component), which kinematically overlap one another in a way that makes it difficult (if not impossible) to find selection criteria capable of disentangling them. This realization pushed us to drop the searching for better selection criteria and led us to look for an alternative approach.

The novelty of this work is the introduction of the analysis of the $e-Z_{\max }$ plane ${ }^{8}$, which permits us to group stars having similar orbits. In fact, eccentricity determines the shape of the orbit, and $Z_{\max }$ determines the oscillation amplitude of the stars in the Galactic plane, i.e., the probability to find the star in the region typically occupied by the thick disc. We applied this analysis to the SN60 sample, and divided it into nine groups on the $e-Z_{\max }$ plane. By studying the distributions of $R_{\mathrm{m}}, R_{\mathrm{p}}, V_{\text {rot }}$, $[\mathrm{Fe} / \mathrm{H}]$, and $[\alpha / \mathrm{Fe}]$ of these groups, we found stellar samples that identify the thin and thick discs nicely, as well as a third sample lacking a clear thin- thick-disc classification.

In particular, we identified an interesting subsample of stars with high eccentricities $(0.4<e<0.6)$, low $Z_{\max }$ (below $1 \mathrm{kpc}$ ), with guiding radii in the inner disc $\left(R_{\mathrm{p}} \sim 3 \mathrm{kpc}\right.$ and $R_{\mathrm{m}} \sim$ $6 \mathrm{kpc})$, and with broad distributions in $[\mathrm{Fe} / \mathrm{H}]$ and $[\alpha / \mathrm{Fe}]$. The $[\mathrm{Fe} / \mathrm{H}]$-poor tail of the distribution is composed of stars that have properties similar to thick disc stars, whereas the high $[\mathrm{Fe} / \mathrm{H}]$ tail shares with them the kinematical signature but differs clearly in Fe abundance, as well as in $\alpha$ enhancement $([\alpha / \mathrm{Fe}])$. A sample of stars with similar orbits and different metallicities suggests the existence of a heating/migration mechanism that pushes stars from the inner part of the Galaxy outward. However, we cannot discard the possibility that such stars have been kinematically heated by merging events or that they belong to a merged satellite themselves (see e.g. Helmi et al. 2006).

Our results support a number of previous works that have shown that the thin and thick discs overlap kinematically. Even if a clear separation did exist in the past, heating from spiral arms (e.g., Carlberg \& Sellwood 1985; Minchev \& Quillen 2006), a central bar (e.g., Minchev \& Famaey 2010), and giant molecular clouds (e.g., Jenkins \& Binney 1990) would have blurred the kinematical borders of the two discs and finally merged them. Additionally, radial migration due to transient spiral density waves (Sellwood \& Binney 2002), the interaction among multiple spirals (Minchev \& Quillen 2006) or bar and spirals (Minchev \& Famaey 2010; Minchev et al. 2011; Brunetti et al. 2011), and the effect of minor mergers (Quillen et al. 2009; Bird et al. 2011) introduces an even harder problem, since by changing their angular momenta, stars arriving in the solar neighborhood have kinematics that are indistinguishable from those born in-situ. In case of satellite accretion (e.g., Villalobos \& Helmi 2008; Abadi et al. 2003) the kinematical borders would also be blurred (because the consequent kinematical heating of the pre-existing disc) with, in addition, a chemical overlap of the accreted stellar populations to the Galactic population. Highresolution spectroscopic surveys would be needed to distinguish the chemical fingerprints of the extra-galactic population from the one born in situ.

Both stellar heating and migration are time-dependent processes. Therefore, in the hypothesis that originally thin and thick

\footnotetext{
8 A different use of the $e-Z_{\max }$ plane was previously made by Feltzing \& Bensby (2008).
} 
discs were kinematically distinguishable, the natural question arises: How long does it take to delete the original kinematical borders between them? On the basis of our kinematic and chemical data, we infer that this time should be rather long (comparable to the Galaxy's lifetime), given that the RAVE sample contains stars with signs of kinematic diffusion together with the expected thin- and thick-disc populations. Models of our Galaxy, together with tools that create synthetic surveys (Sharma et al. 2011), will be employed in our next work in order to compare observations with up-to-date models of the Milky Way. Qualitative and quantitative comparisons of our data with detailed chemodynamical models that follow the evolution of a Milky Way-like disc for the entire expected thin- and thick-disc lifetimes, are needed to understand the formation and evolution of the Galactic discs better.

Acknowledgements. C.B. thanks J. Binney, A. Just, and B. Anguiano for their useful comments. We acknowledge funding from Sonderforschungsbereich SFB 881 "The Milky Way System" (subproject A5) of the German Research Foundation (DFG). Funding for RAVE has been provided by: the Australian Astronomical Observatory; the Leibniz-Institut fuer Astrophysik Potsdam (AIP); the Australian National University; the Australian Research Council; the French National Research Agency; the German Research Foundation (SPP 1177 and SFB 881); the European Research Council (ERC-StG 240271 Galactica); the Istituto Nazionale di Astrofisica at Padova; The Johns Hopkins University; the National Science Foundation of the USA (AST-0908326); the W.M. Keck foundation; the Macquarie University; the Netherlands Research School for Astronomy; the Natural Sciences and Engineering Research Council of Canada; the Slovenian Research Agency; the Swiss National Science Foundation; the Science \& Technology Facilities Council of the UK; Opticon; Strasbourg Observatory; and the Universities of Groningen, Heidelberg, and Sydney. The RAVE web site is at http://www . rave-survey .org.

\section{References}

Abadi, M. G., Navarro, J. F., Steinmetz, M., \& Eke, V. R. 2003, ApJ, 597, 21 Adibekyan, V. Zh., Santos, N. C., Sousa, S. G., \& Israelian, G. 2011, A\&A, 535, 11

Bensby, T., \& Feltzing, S. 2012, EPJ Web Conf., 19, id. 04001

Bensby, T., Feltzing, S., \& Lundström, I. 2003, A\&A, 410, 527

Bensby, T., Feltzing, S., Lundström, I., \& Ilyin, I. 2005, A\&A, 433, 185

Bijaoui, A., Recio-Blanco, A., de Laverny, P., \& Ordenovic, C. 2012, Statistical methodology, 9, 55

Bird, J. C., Kazantzidis, S., \& Weinberg, D. H. 2011, MNRAS, 2140

Boeche, C., Siebert, A., Williams, M., et al. 2011, AJ, 142, 193

Bovy, J., Rix, H.-W., Liu, C., et al. 2012, ApJ, 753, 148

Bournaud, F., Elmegree, B. G., \& Martig, M. 2009, ApJ, 694, L158

Breddels, M. A., Smith, M. C., Helmi, A., et al. 2010, A\&A, 511, A90

Brook, C. B., Stinson, G., Gibson, B. K., et al. 2012, MNRAS, 419, 771

Brunetti, M., Chiappini, C., \& Pfenniger, D. 2011, A\&A, 534, A75

Burnett, B., Binney, J., Sharma, S., et al. 2011, A\&A, 532, A113

Carlberg, R. G., \& Sellwood, J. A. 1985, ApJ, 292, 79

Casagrande, L., Schönrich, R., Asplund, M., et al. 2011, A\&A, 530, A138

Chen, J. Y., Rockosi, C. M., Morrison, H. L., et al. 2012, ApJ, 746, 149

Chiappini, C. 2009, The Galaxy Disk in Cosmological Context, Proc.

International Astronomical Union, eds. J. Andersen, J. Bland-Hawthorn, \&

B. Nordström, IAU Symp., 254, 191

Dehnen, W., \& Binney, J. 1998a, MNRAS, 294, 429

Dehnen, W., \& Binney, J. J. 1998b, MNRAS, 298, 387

Di Matteo, P., Qu, Y., Lehnert, M. D. \& van Driel, W. 2012, Assembling the Puzzle of the Milky Way, Le Grand-Bornand, France, eds. C. Reylé, A. Robin, \& M. Schultheis, EPJ Web of Conf., 19, 4002

Feltzing, S., \& Bensby, T. 2008, Phys. Scr., 133, 014031
Forbes, J., Krumholz, M. R., \& Burkert, A. 2012, ApJ, 754, 48

Fuhrmann, K. 1998, A\&A, 338, 161

Fuhrmann, K. 2008, MNRAS, 384, 173

Jenkins, A., \& Binney, J. 1990, MNRAS, 245, 305

Gratton, R., Carretta, E., Matteucci, F., \& Sneden, C. 1996, ASPC, 92, 307

Gratton, R. G., Carretta, E., Matteucci, F., \& Sneden, C. 2000, A\&A, 358, 671

Gratton, R. G., Carretta, E., Desidera, S., et al. 2003, A\&A, 406, 131

Grenon, M. 1999, Astrophys. Space Sci., 265, 331

Guedes, J., Callegari, S., Madau, P., \& Mayer, L. 2011, ApJ, 742, 76

Helmi, A., Navarro, J. F., Nordström, B., et al. 2006, MNRAS, 365, 1309

Karataş, Y., \& Klement, R. J. 2012, NewA, 17, 22

Kordopatis, G., Recio-Blanco, A., de Laverny, P., et al. 2011a, A\&A, 535, A106

Kordopatis, G., Recio-Blanco, A., de Laverny, P., et al. 2011b, A\&A, 535, A107

Lee, Y. S., Beers, T. C., \& Sivarani, T. 2008a, AJ, 136, 2050

Lee, Y. S., Beers, T. C., \& Sivarani, T. 2008b, AJ, 136, 2022

Lee, Y. S., Beers, T. C., Deokkeun, A., et al. 2011, AJ, 738, 187

Liu, C., \& van de Ven, G. 2012, MNRAS, 425, 2144

Marigo, P., Girardi, L., Bressan, A., et al. 2008, A\&A, 482, 883

Matijevič, G., Zwitter, T., Bienaymé, O., et al. 2012, ApJS, 200, 14

Minchev, I., \& Famaey, B. 2010, ApJ, 722, 112

Minchev, I., \& Quillen, A. C. 2006, MNRAS, 368, 623

Minchev, I., Famaey, B., Combes, F., et al. 2011, A\&A, 527, A147

Minchev, I., Famaey, B., Quillen, A. C., \& Dehnen, W. 2012a, EPJ Web Conf., 19, id. 07002

Minchev, I., Famaey, B., Quillen, A. C., et al. 2012b, A\&A, 548, A126

Minchev, I., Famaey, B., Quillen, A. C., et al. 2012c, A\&A, 548, A127

Minchev, I., Chiappini, C., \& Martig, M. 2012d, A\&A, submitted [arXiv: 1208.1506]

Navarro, J. F., Abadi, M. G., Venn, K. A., Freeman, K. C., \& Anguiano, B. 2011, MNRAS, 412, 1203

Nissen, P. E., \& Schuster, W. J. 2010, A\&A, 511, L10

Nordström, B., Mayor, M., Andersen, J., et al. 2004, A\&A 418, 989

Pasetto, S., Grebel, E. K., Zwitter, T., et al. 2012a, A\&A, 547, A71

Pasetto, S., Grebel, E. K., Zwitter, T., et al. 2012b, A\&A, 547, A70

Pilkington, K., Few, C. G., Gibson, B. K., et al. 2012, A\&A, 540, A56

Piontek, F., \& Steinmetz, M. 2011, MNRAS, 410, 2625

Quillen, A. C., Minchev, I., Bland-Hawthorn, J., \& Haywood, M. 2009, MNRAS, 397, 1599

Quinn, P. J., Hernquist, L., \& Fullagar, D. P. 1993, ApJ, 403, 74

Recio-Blanco, A., Bijaoui, A., \& de Laverny, P. 2006, MNRAS, 370, 141

Reddy, B. E., Lambert, D. L., \& Allende Prieto, C. 2006, MNRAS, 367, 1329

Roškar, R., Debattista, V. P., Quinn, T. R., Stinson, G. S., \& Wadsley, J. 2008, ApJ, 684, L79

Sánchez-Blázquez, P., Courty, S., Gibson, B. K., \& Brook, C. B. 2009, MNRAS, 398,591

Scannapieco, C., Wadepuhl, M., Parry, O. H., et al. 2011, MNRAS, 423, 1726

Schlesinger, K. J., Johnson, J. A., Rockosi, C. M., et al. 2012, ApJ, 761, 160

Schuster, W. J., Parrao, L., \& Contreras Martinez, M. E. 1993, A\&AS, 97, 951

Schoenrich, R., \& Binney, J. 2009a, MNRAS, 396, 203

Schönrich, R., \& Binney, J. 2009b, MNRAS, 399, 1145

Sellwood, J. A., \& Binney, J. J. 2002, MNRAS, 336, 785

Sharma, S., Bland-Hawthorn, J., Johnston, K. V., \& Binney, J. 2011, ApJ, 730, 3

Siebert, A., Williams, M. E. K., Siviero, A., et al. 2011, AJ, 141, 187

Spagna, A., Lattanzi, M. G., Re Fiorentin, P., \& Smart, R. L. 2010, A\&A, 510, L4

Steinmetz, M. 2012, AN 5/6, 523

Steinmetz, M., Zwitter, T., Siebert, A., et al. 2006, AJ, 132, 1645

Stinson, G., Brook, C., Prochaska, J. X., et al. 2012, MNRAS, 425, 1270

Teuben, P. J. 1995, The Stellar Dynamics Toolbox NEMO, in Astronomical Data Analysis Software and Systems IV, eds. R. Shaw, H. E. Payne, \& J. J. E. Hayes, PASP Conf. Ser., 77, 398

Trevisan, M., Barbuy, B., Eriksson, K., et al. 2011, A\&A, 535, A42

Veltz, L., Bienaymé, O., Freeman, K. C., et al. 2008, A\&A, 480, 753

Villalobos, A., \& Helmi, A. 2008, MNRAS, 391, 1806

Wilson, M. L., Helmi, A., Morrison, H. L., et al. 2011, MNRAS, 413, 2235

Yanny, B., Rockosi, C., Newberg, H. J., et al. 2009, AJ, 137, 4377

Zwitter, T., Siebert, A., Munari, U., et al. 2008, AJ, 136, 421

Zwitter, T., Matijevič, G., Breddels, M. A., et al. 2010, A\&A, 522, A54 